\title{
„Sie alle, Sie sind die Heldinnen und Helden \\ in der Corona-Krise.“ \\ Heldentum in Zeiten der Pandemie
}

Daniel Koch (Kassel)

\begin{abstract}
In 2020, the topic "Corona" will be omnipresent in the media. The headlines are dominated by negative reports about death rates, exit restrictions and the economic downturn. However, there are also numerous encouraging reports about "Corona heroes" who are contributing to overcoming the crisis with their self-sacrificing commitment. But it is not only journalists who draw attention to their deeds. On Twitter, Instagram and Co., politicians, companies and private individuals also thank the "heroes of everyday Corona life". These positive exceptional figures are examined in more detail in the article, with the focus on the communicative patterns of their staging. For a specific use of language does not simply depict reality unchanged, but always provides a perspective on reality. Thus, the investigation not only reveals insights into language, but also provides information about how our society deals with critical events. Because heroes - so the conviction - solve crises. They perform heroic deeds with which they alleviate the needs and worries of the people. At the same time, they show us what values and behaviour are necessary to cope with a delicate situation. Overall, the analyses show that "heroes" have been omnipresent since the beginning of the pandemic at the latest - and they are often portrayed in the style of superheroes. The fact that Germany is repeatedly described as "post-heroic" is difficult to understand against this background. Rather, we seem to live in a "superheroic society".
\end{abstract}

\section{$1 \quad$ Einleitung}

In Talkshows und im Feuilleton wird Deutschland immer wieder als ,,postheroische Gesellschaft" beschrieben. Diese Vorstellung wurde insbesondere von Herfried Münkler (2006, 2007, 2015) geprägt, der anführt, „Helden“ und die kriegerischen und aufopferungsvollen Ideale, für die sie stehen, hätten nach dem Ende des Zweiten Weltkriegs massiv an Bedeutung verloren. Aus „heroischen“ seien so „postheroische Gesellschaften“ geworden (Münkler 2007: 749). Zweifellos richtig ist, dass klassische KRIEGERHELDEN ${ }^{1}$ ihren Status als gesellschaftliche Leitfiguren zu großen Teilen eingebüßt haben. Doch anders als der Begriff ,postheroische Gesellschaft" suggeriert, sind HELD*INNEN auch heute noch im öffentlichen Diskurs sehr präsent. So

\footnotetext{
${ }^{1}$ Durch Kapitälchen wird auf das Konzept HeLD verwiesen, das Wort Held wird hingegen kursiv gesetzt.
} 
begegnen sie uns etwa in den Reden von Politiker*innen, in der Sportberichterstattung, in der Werbung, in Filmen und in Videospielen. Mit der Ausbreitung von Covid-19 rücken 2020 zudem CORONA-HELD*INNEN verstärkt in den Fokus der Öffentlichkeit, wie die folgenden Überschriften von Nachrichtenartikeln belegen:

Coronakrise in Deutschland: Sie sind Helden des Alltags

Die Coronahelden - Diese Menschen halten Deutschland am Laufen

Einkaufshelden für Sie in Staufenberg unterwegs

Corona: Die Helden des Krisenalltags verdienen unseren Dank

Berlin stellt Corona-Helden Bonus in Aussicht

FCN-Ehrentrikot für Helden in Krankenhäusern und Supermärkten
(Mundt 2020)

(Bild.de 2020a)

(Einkaufshelden-Team 2020)

(Clemens 2020)

(n-tv 2020)

(1. FC Nürnberg 2020)

Die zahlreichen HELD*INNEN unserer modernen Medienlandschaft legen den Schluss nahe, dass wir weniger in einer ,postheroischen“, sondern vielmehr in einer ,superheroischen Gesellschaft“ leben (Koch 2020: 308-335): HELD*INNEN sind im öffentlichen Diskurs nicht nur omnipräsent, sondern werden häufig auch mit Elementen einer Superheldenikonografie inszeniert (dazu später mehr). Da insbesondere in unsicheren Zeiten die Menge von Heroisierungen oft zunimmt, ist eine kritische Auseinandersetzung mit den HELD* INNEN der ,superheroischen Gesellschaft" unerlässlich. Denn

Sprache ist kein neutrales Medium, das die Gegenstände und Sachverhalte „unverändert“, in ihrem Ursprung oder gar 1:1 ins Bewusstsein der Menschen bringt. Vielmehr werden die gesamtgesellschaftlich relevanten Wissensbestände durch die eingesetzten sprachlichen Mittel (mit)geformt. Wem es beispielsweise gelingt, bestimmte Bezeichnungen und Ausdrucksweisen in Diskursen durchzusetzen oder bestimmten sprachlichen Mustern spezifische Bedeutungsaspekte zuzuschreiben und diese im öffentlichen Bewusstsein zu verankern, der prägt Deutungen von Sachverhalten mit. Sprache erzeugt die fachlichen Gegenstände und Sachverhalte allererst selbst, sprachliche Mittel und Formen konstituieren das Wissen.

(Felder 2009: 11)

Vor diesem Hintergrund wird in dem Beitrag dargestellt, mit welchen kommunikativen Mitteln „normale“ Menschen während der Corona-Pandemie zu Held*innen erhöht werden. Dabei werden Überlegungen aus der Dissertation des Autors (Koch 2020) aufgegriffen und ausgeführt. Grundlage der Untersuchungen ist eine Auswahl von Heroisierungen, die während des ersten Lockdowns im Zeitraum von Mitte März bis Ende April 2020 entstanden sind. Analysiert werden schrift-sprachlichen Heldendarstellungen seitens der Politik (Steinmeier 2020; Bundesministerium der Finanzen 2020) und der Presse (Würth 2020; Wellerdiek/Witzig 2020; Bennet/Berenson 2020; Bild.de 2020a, 2020b; Clemens 2020; focus.de 2020; Hannoversche Allgemeine 2020; Liebold 2020; Link 2020; Mundt 2020; n-tv 2020; Späth 2020a, 2020b; spiegel.de 2020a, 2020b; STIMME.de 2020; Töpper 2020; Witterauf 2020; ZDF 2020a, 2020b). Daneben werden aber auch multimodale Quellen aus den sozialen Medien (Edeka 2020; Netto MarkenDiscount 2020; Lidl Deutschland 2020; myStipendium.de 2020; nvvbewegt 2020) in das Korpus aufgenommen, denn

[m] it Blick auf die Allgegenwärtigkeit und Unvermeidbarkeit des Gebrauchs unterschiedlicher Zeichenressourcen im Rahmen der Kommunikation muss gelten: Der Sprachgebrauch einer Gemeinschaft lässt sich nur auf der Basis einer möglichst umfassenden Kontextualisierung angemessen erfassen, also dann, wenn Sprache in ihre relevanten Deutungs- bzw. Verstehensrahmen 
eingebettet wird. [...] Eine an kulturellen Phänomenen interessierte gebrauchsorientierte Sprachwissenschaft, die einen Beitrag zur „Analyse gesellschaftlichen Wissens“ leisten will (Busse 2000), sollte sich deshalb einer multimodalen Sprachbeschreibung öffnen.

(Klug/Stöck1 2015: 243)

Aufgrund der kulturwissenschaftlichen Ausrichtung des Beitrags fördern die linguistischen Untersuchungen nicht nur Erkenntnisse über Sprache zutage, sondern sie geben auch Auskunft über den Umgang unserer Gesellschaft mit kritischen Ereignissen. Denn Held*innen - so die Überzeugung - lösen Krisen. Sie vollbringen „Heldentaten“, mit denen sie die Nöte und Sorgen der Menschen lindern. Gleichzeitig leben sie uns vor, welche Werte und Verhaltensweisen nötig sind, um eine heikle Situation zu bewältigen. Diese Orientierungs- und Vorbildfunktion von Held*innen ist untrennbar mit dem Hochwertwort Held verbunden und hat sich zu dessen deontischer Bedeutung verdichtet.

Die deontischen Bedeutungskomponenten sind die lexikalische Entsprechung dessen, was sich mentalitätsgeschichtlich als das kollektive Sollen (also auch als kollektives Wollen) darstellt. Kraft der in den Wörtern mitgemeinten und mitausgesagten Sollenskomponenten der Bedeutung - eben der „deontischen Bedeutung“ - sind die Wörter die Vehikel oder Abbreviaturen von Gedanken auch bezüglich dessen, was der Fall sein soll und nicht allein bezüglich dessen, was der Fall ist.

(Hermanns 2012: 20, Hervorheb. i. O.)

Aufgrund ihrer deontischen Bedeutungen ist die „Aufforderung zum Tun oder Lassen [...] bei Schlagwörtern [...] also im Lexem selbst angelegt" (Gardt 2012: 24).

Die deontischen Bedeutungskomponenten des Wortes Held fordern implizit dazu auf, Held*innen grundsätzlich positiv gegenüberzustehen, sie zu unterstützen und zu bewundern und ihrem herausragenden Beispiel nachzueifern. Bei jeder Nutzung des Hochwertwortes werden diese positiv deontischen Komponenten ,mitbedeutet“. Durch die Zuschreibung eines Heldenstatus wird daher der oder die Heroisierte als eine herausragende Persönlichkeit charakterisiert, zu der es aufzuschauen gilt. Hieraus ergeben sich zwei Konsequenzen. Zum einen wird Heldentum als erstrebenswert wahrgenommen und die Aussicht darauf, selbst „Held“ zu werden, kann handlungsleitend wirken. Zum anderen sind Zuschreibungen von „Heldentum“ eine Möglichkeit, verdiente Personen in das öffentliche Bewusstsein zu rücken und ihnen Dank und Anerkennung auszusprechen. Daher werden seit Beginn der Corona-Pandemie verstärkt Menschen in „systemrelevanten" Berufen heroisiert, deren Engagement für das Funktionieren der Gesellschaft unerlässlich ist. Zwar handelt es sich bei Heroisierungen von Corona-Held*innen um ein Krisenphänomen, jedoch wird dabei kein gänzlich neuer Heldentypus erschaffen. Stattdessen wird an den bereits etablierten Typus der Alltagsheld*innen angeknüpft. Auf diese Weise wird die Integration der Corona-Held*innen in das „,heldische“ Vorwissen der Rezipient*innen erleichtert, was wiederum deren Wahrnehmung als Held*innen befördert. Anhand eines Auszugs einer Rede von Frank-Walter Steinmeier vom 26.03.2020 sollen einige der zentralen Charakteristika dieser beiden Heldenbilder beleuchtet werden. 


\section{Wie sich Corona-Held*innen in unser „heldisches“ Vorwissen einfügen}

\subsection{Corona-Held*innen als spezielle Ausprägung des Typus der Alltagsheld*innen}

Viele, sehr viele von Ihnen helfen jetzt; gehen für ihre älteren Nachbarn einkaufen; hängen Tüten mit Lebensmitteln für Obdachlose an ,Gabenzäune'; und über 40.000 von Ihnen haben am Wochenende virtuell die Köpfe zusammengesteckt und hunderte kreative Lösungen für die Sorgen und Nöte dieser Krisenzeit entwickelt.

Sie alle, Sie sind die Heldinnen und Helden in der Corona-Krise. Einige von Ihnen habe ich in dieser Woche am Telefon gesprochen: die Filialleiterin zum Beispiel im Supermarkt, die mit ihrem Team 12-Stunden-Schichten schiebt, damit die Regale nicht leer bleiben. Oder die Grundschullehrerin, die auf dem Dachboden Lernvideos für ihre Schülerinnen und Schüler dreht. Die Sozialarbeiterin, die eine Telefonkette für Menschen organisiert, die sich einsam fühlen. Und der Arzt, die Pflegerin, die Apothekerin, die jenseits der Erschöpfung ihren lebensrettenden Beruf verrichten.

(Steinmeier 2020)

CORONA-HELD*INNEN fügen sich in den zivil-demokratischen Wertekanon unserer Gesellschaft ein. Klassische Beschränkungen zur Erlangung eines Heldenstatus wie eine elitäre Abstammung, das Kriegerdasein, außerordentliche Fähigkeiten oder das männliche Geschlecht sind daher keine konstitutiven Merkmale dieser HELD*INNEN (zu dieser „Demokratisierung von Heldentum“ cf. Koch, 2020: 145-152). Vielmehr stammen die CORONA-HELD*INNEN - wie die meisten ALLTAGSHELD*INNEN - aus allen gesellschaftlichen Schichten und Berufsgruppen (Angestellte im Supermarkt, Lehrerin, Sozialarbeiterin, Arzt, Pflegerin, Apothekerin). So unterschiedlich wie die CORONA-HELD*INNEN so verschieden sind auch deren Heldentaten (einkaufen, entwickeln, Regale befüllen, lehren, betreuen, Leben retten). Obwohl sich diese „Heldentaten" augenscheinlich immens voneinander unterscheiden, haben sie dennoch eine zentrale Gemeinsamkeit: Sie kommen dem Wohle der Gemeinschaft zugute. Diese Ausrichtung spiegelt sich anschaulich im häufigen Gebrauch zweier sprachlicher Muster wider. Zum einen wird oft auf ein Sprichwort referiert und angeführt, die CORONA-HELD*INNEN halten „die Gesellschaft“ (spiegel.de 2020b), „das System“ (STIMME.de 2020), „,eine Stadt“ (Würzer 2020) oder generell „den Laden am Laufen“ (Lukesch 2020: 5). Zum anderen verweist auch die syntagmatische Struktur der Beschreibungen ihrer „Heldentaten“ auf den Dienst an der Gemeinschaft. Diese werden häufig mit der Präposition für eingeleitet, an die sich die Nennung einer hilfsbedürftigen Personengruppen anschließt (,für ihre älteren Nachbarn“; „für Obdachlose“; „für ihre Schülerinnen und Schüler“; „Telefonkette für Menschen organisiert, die sich einsam fühlen“). Diese syntagmatische Struktur findet sich seit Jahrzehnten in Heroisierungen verschiedenster Heldentypen. Aufgrund ihrer Konventionalität im Heldendiskurs hat sie maßgeblich unsere Vorstellung von HELDENTUM beeinflusst und bewirkt, dass wir heute von HELD*INNEN erwarten, dass sie sich für die Gemeinschaft einsetzen. Dementsprechend sind auch keine herausragenden und außergewöhnlichen Leistungen (mehr) notwendig, um HELD*IN zu werden. Stattdessen können bereits kleine Akte der alltäglichen Hilfeleistung einen ,normalen“ Menschen zu einem „Helden“ machen, wie etwa im Teaser der fortlaufenden spiegel.de-Rubrik „Heldinnen und Helden des Corona-Alltags“" ausgeführt wird: 
In der aktuellen Zeit braucht es Leute, die im Großen und im Kleinen mit anpacken, damit unsere Gesellschaft weiter funktioniert - sei es durch ihr berufliches oder privates Engagement. Wir stellen vor: Die Heldinnen und Helden des Corona-Alltags.

(spiegel.de 2020a)

CORONA-HELD*INNEN sorgen durch ihr Engagement „,im Großen und Kleinen“ dafür, dass unsere Gesellschaft während der Pandemie weiter funktioniert. In dieser Darstellung manifestiert sich das im Alltagsheldendiskurs sehr etablierte Motiv des die-Welt-im-Kleinen-besser-Machens. Wie die beschriebene syntagmatische Struktur der Beschreibung der Heldentat trägt auch dieses sprachliche Muster dazu bei, dass unsere HELD*INNEN immer ziviler (im Sinne von nicht-militärisch) werden und wir HELDENTUM zunehmend im Alltag verorten.

\subsection{Corona-Held*innen als Kämpfer*innen}

Insbesondere ihre zivile Ausrichtung unterscheidet viele aktuelle von älteren Heldendarstellungen, in denen oft primär herausragende Einzelpersönlichkeiten für ihren aufopferungsvollen Kampf gegen einen Feind heroisiert wurden. Die meisten ALLTAGSHELD*INNEN sind mit einer derartig kriegerisch geprägten Vorstellung von HELDENTUM jedoch nur schwer zu vereinbaren, weshalb nicht alle Rezipient*innen eine kohärente Verbindung zwischen diesen „,normalen“ Menschen und ihren prototypischen HELD*INNEN herstellen können. ALLTAGSHELD*INNEN (und andere Heldentypen) fügen sich nicht in ihr bestehendes Heldenbild ein und werden dementsprechend auch nicht als solche wahrgenommen. Anders als die meisten ALLTAGSHELD*INNEN weisen jedoch einige CORONA-HELD*INNEN eine kriegerisch-agonale Prägung auf, was sie in Tradition zu etablierten Heldenfiguren stellt und damit auch ihre Wahrnehmung als ,echte“ HELD*INNEN befördert. Diese kriegerische Ausrichtung von CORONA-HELD*INNEN steht im Zusammenhang mit der über die Grenzen von Einzelstaaten hinaus zu verzeichnenden Praxis, den Umgang mit dem Virus als einen Kampf zu konzeptualisieren. Emmanuel Macron spricht etwa vom „guerre sanitaire“ (Süddeutsche Zeitung Online 2020), Donald Trump vom „medical war” (Bennet/Berenson 2020) und das deutsche Bundesfinanzministerium hat im „Kampf gegen Corona“ (Bundesministerium der Finanzen 2020) einen „Corona-Schutzschild“ (ibd.) für die heimische Wirtschaft entworfen. Innerhalb dieses Narrativs vom Kampf gegen das CoronaVirus kommt einigen CORONA-HELD*INNEN die Rolle als Kämpfer*innen zu:

Apotheker arbeiten in der Coronakrise an vorderster Front und haben täglich mit Herausforderungen zu kämpfen - von der Gefahr einer Ansteckung bis hin zu Lieferengpässen.

(ZDF 2020a, Hervorheb. D. K.)

Das Leben in Deutschland scheint weitgehend stillzustehen. Geschäfte und Restaurants haben geschlossen. Ärzte, Pflegekräfte, Lkw-Fahrer - BamS traf die Helden der Stunde, die trotz der Corona-Krise die Stellung halten. [...] Kunden stürmen in ihren Laden, reißen sich um Klopapier und Desinfektionsmittel. Doch Melinda Angsten bleibt inmitten der Corona-Hysterie standhaft. [...] ,Ich sitze an der Kasse, räume Regale ein, mache die Dienstpläne. Auf Urlaub verzichten wir alle erst mal, aber mein Team versteht das. Immerhin stehen wir hier an vorderster Front.“

(Bild.de 2020a, Hervorheb. D. K.)

Durch die Verwendung von Elementen des semantischen Feldes „Militärischer Kampf“ (an vorderster Front; mit Herausforderungen kämpfen; die Stellung halten; stürmen; standhaft blei- 
ben) werden die CORONA-HELD*INNEN in diesen beiden Texten als Kämpfer*innen konzeptualisiert, was sie in die Nähe von tradierten Heldenfiguren rückt. Dass der „Kampf“ gegen eine Krankheit heroisiert und im Zuge dessen ein militärisch geprägter Wortschatz genutzt wird, ist keineswegs ein neues Phänomen (cf. hierzu und den folgenden Ausführungen Koch, 2020: 275-284). Generell finden sich im Krankheitsdiskurs sehr häufig Aussagen, wie „den Krebs besiegen, bekämpfen, unterliegen, den Kampf gegen eine Krankheit ausfechten, aufgeben“ etc. Diese Praxis der Heroisierung eines „Kampfes“ gegen eine Krankheit kann positive Effekte auf die Psyche schwer Erkrankter haben. Denn durch die Konzeptualisierung als Kämpfer*innen wird ihnen ein aktiver Einfluss auf ihren Krankheitsverlauf attestiert. Diese Zuschreibung von Handlungsmacht kann bei den Betroffenen die Einsicht vermitteln, dass ein beherzter „Kampf“ gegen die Krankheit ihre Genesung positiv beeinflusst. Mit dem Ausbruch der Corona-Pandemie ist plötzlich die gesamte Welt von den unmittelbaren und einschneidenden Auswirkungen der Krankheit betroffen. Daher kann auch die Umdeutung des Leidens unter dem Corona-Virus zu einem aktiven „Kampf“ gegen die Krankheit der Gesellschaft helfen, die Maßnahmen zur Eindämmung der Pandemie zu ertragen.

Neben diesem positiven Effekt auf die kollektive Psyche erfüllen die Heroisierungen von CORONA-HELD*INNEN auch eine gemeinschaftsstiftende Funktion. Denn der „Kampf“ gegen einen gemeinsamen äußeren „Feind“ Corona verbindet. Dieser innere Zusammenhalt ist essenziell, da jede*r Einzelne für die Ausbreitung der Krankheit mitverantwortlich ist. Aus diesem Grund nutzen auch zahlreiche CORONA-HELD*INNEN in medizinischen Berufen ihre Stellung als gesellschaftliche Leitfiguren und appellieren an die Bevölkerung, sie in ihrem „Kampf“ gegen die Krankheit zu unterstützen:

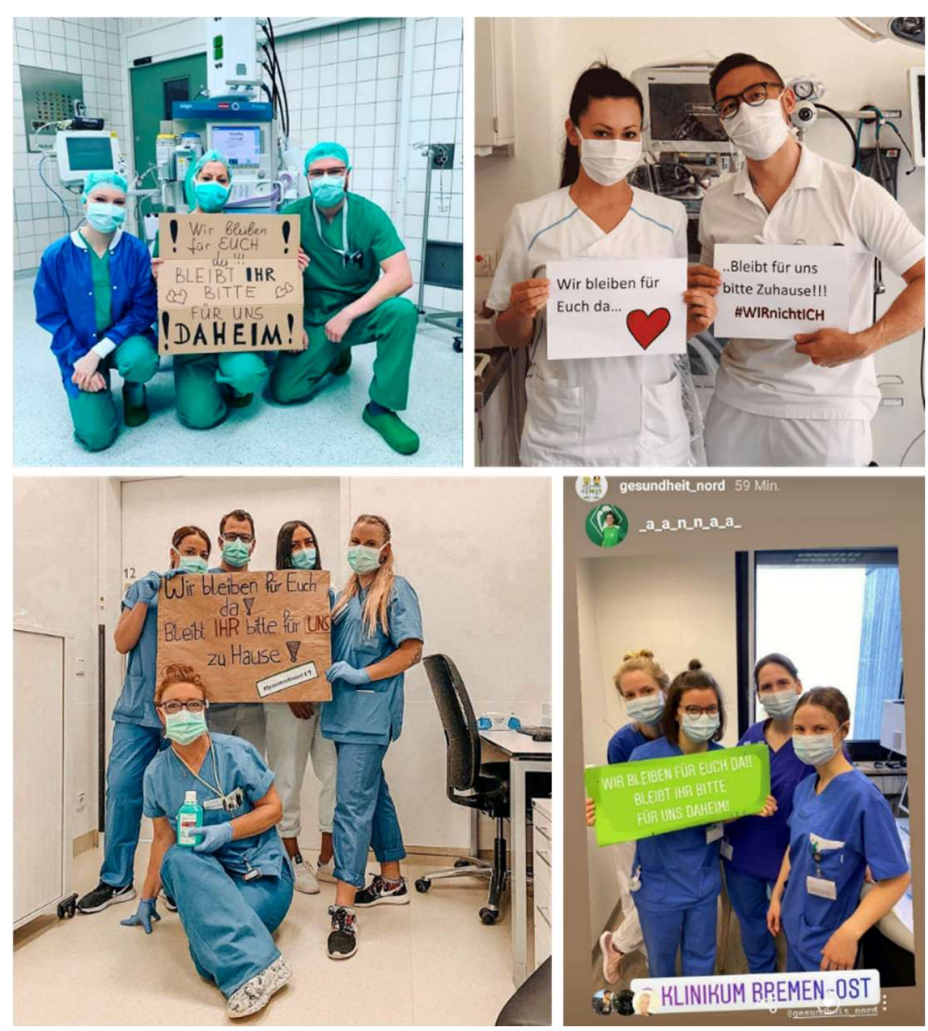

Abbildung 1: Corona-Held*innen aus medizinischen Berufen rufen auf, zu Hause zu bleiben (spiegel.de 2020a) 
„Wir bleiben für Euch da! Bleibt Ihr bitte für uns zu Hause!“ Mit diesem Appell richten sich Ärzt*innen und Pfleger*innen in den sozialen Medien an die Bevölkerung. Zwar handelt es sich hierbei nicht um klassische (Selbst-)Heroisierungen, dennoch können auch diese Darstellungen die Wahrnehmung von Mediziner*innen und Pfleger*innen als HELD*INNEN begünstigen, da sich in ihnen tradierte und neuere Vorstellungen von HELDENTUM verdichten. So sind etwa auf der bildlichen Ebene Elemente des semantischen Feldes „Medizin“ sehr präsent (medizinisches Gerät im Hintergrund, Krankenhausbekleidung, Mundschutz). Zwar handelt es sich hierbei um eine typisch zivile Domäne, doch da der Umgang mit einer Krankheit oft als ein Kampf konzeptualisiert wird, können die Rezipient*innen dem medizinischen Personal die Rolle von Kämpfer*innen zuschreiben und sie in Tradition zu kriegerischen HELD*INNEN stellen. Dieser Zuschreibungsprozess wird zusätzlich durch die Kleidung der HELD*INNEN befördert. Denn ähnlich wie Soldat*innen tragen die Mediziner*innen eine standardisierte „Uniform".

Wie in den meisten Heroisierungen von ALLTAGSHELD*INNEN wird hier nicht zwischen einem männlich-kämpfenden und einem weiblich-helfenden HELDENTUM unterschieden. So sind sowohl Männer als auch Frauen auf den Darstellungen vertreten und auf der sprachlich-textuellen Ebene wird der Einsatz der Mediziner*innen für die Gemeinschaft betont (,Wir bleiben für euch da“). Hieran schließt sich die Forderung der CORONA-HELD*INNEN an (,Bleibt ihr bitte für uns daheim“). In beiden Sätzen findet sich die für Alltagsheldendarstellungen typische syntagmatische Struktur aus der Präposition für, auf die eine hilfsbedürftige Gruppe folgt (euch bzw. uns). Doch trotz ihrer zivilen Prägung rücken auch diese beiden Sätze die CORONAHELD*INNEN in die Nähe von KRIEGERHELDEN, da sie eine Abwandlung des Leitspruchs der Musketiere aus Alexandre Dumas Roman Die drei Musketiere sind (,Einer für alle, alle für einen."). In dieser Gegenüberstellung von „,wir für euch, ihr für uns“ manifestiert sich zudem die gegenseitige Abhängigkeit von HELD*INNEN und ihrer Gesellschaft: HELD*INNEN agieren zum Wohle der Gemeinschaft, dieser Aufgabe können sie jedoch nur nachkommen, wenn sie dabei von der gesamten Bevölkerung unterstützt werden. Durch derartige kommunikative Muster wird der „Kampf“ gegen das Corona-Virus als eine gesamtgesellschaftliche Aufgabe charakterisiert. Innerhalb dieses Narrativs kann jede*r Einzelne HELD*IN werden, der oder die mit seinem oder ihrem verantwortungsbewussten und solidarischen Handeln zur Bewältigung dieser Ausnahmesituation beiträgt.

\subsection{Corona-Held*innen sind bereit, sich für die Allgemeinheit aufzuopfern}

Vor diesem Hintergrund finden sich neben den in den sozialen Medien verbreiteten Appellen von CORONA-HELD*INNEN aus medizinischen Berufen auch zunehmend Heroisierungen, die explizit dazu auffordern, selbst zu CORONA-HELD*INNEN zu werden. Mitunter werden hierfür sogar materielle Vergütungen in Aussicht gestellt, wie etwa von der Plattform myStipendium.de, die ein Stipendium für „Corona-Helden“ vergibt:

Mehr denn je kommt es aktuell darauf an, dass wir alle miteinander anpacken, helfen und Verantwortung übernehmen. In Zeiten der Corona-Krise gehen viele Menschen weit über sich hinaus und zeigen, dass man als Held keine übernatürlichen Kräfte braucht. Diese Helden wollen wir unterstützen. Daher suchen wir Kämpfer, die ihre Zeit, ihre Energie und ihre Gesundheit für ihre Mitmenschen geben. Wir suchen Menschen, die in diesen schweren Zeiten für ihre Mitmenschen da sind. 
Gefördert werden engagierte Studierende, die mit ihren enormen Einsatz in der Corona-

Krise ihren Mitmenschen helfen. myStipendium möchte daher einen besonderen Helden/Heldin fördern, der die Welt zu einem besseren Ort macht.

\subsection{0 $€$ für Corona-Helden!}

(myStipendium.de 2020, Hervorheb. i. O.)

In dieser Ausschreibung werden die „Corona-Helden“ mit etablierten kommunikativen Mustern des Alltagsheldendiskurses heroisiert. Dies tritt besonders anschaulich in der Darstellung ihrer „Heldentaten“ hervor. Diese werden mit Wörtern des semantischen Feldes „Hilfe/Engagement" (anpacken; helfen und Verantwortung übernehmen) beschrieben und auch die bekannte syntagmatische Struktur aus der Präposition ,für“, auf die eine hilfsbedürftige Gruppe folgt („,ür ihre Mitmenschen“), ist sehr präsent. Weiterhin wird explizit betont, „,dass man als Held keine übernatürlichen Kräfte braucht“ und dass das Engagement dieser HELD*INNEN „die Welt zu einem besseren Ort macht". Obwohl auf diese Weise das HeldENTUM der CORONAHELD*INNEN im Alltag verortet wird, werden ihre Taten gleichzeitig als herausragende Leistungen gewertet. So wird angeführt, diese gingen ,weit über sich hinaus“ und würden ,mit ihren enormen Einsatz in der Corona-Krise ihren Mitmenschen helfen". Innerhalb dieser Heroisierung wird insbesondere gemeinschaftlich orientiertes Verhalten und solidarischem Handeln als heldenhaft und damit nachahmenswert charakterisiert. Hierzu trägt auch die ausgeprägte Nutzung von Wörtern aus dem semantischen Feld „Gemeinschaft" bei (wir alle miteinander; wir; Mitmenschen).

Neben diesen konventionalisierten sprachlichen Mustern des Alltagsheldendiskurses, finden sich auch tradierte Heroisierungsformen. Hierzu zählen etwa die Konzeptualisierung der HELD*INNEN als Kämpfer*innen sowie die Verweise auf deren Opferbereitschaft („Daher suchen wir Kämpfer, die ihre Zeit, ihre Energie und ihre Gesundheit für ihre Mitmenschen geben."). Insbesondere die Betonung ihrer Opferbereitschaft ist zentral für ihre Wahrnehmung als HELD*INNEN und für die Ausweitung des Heldenstatus auf große Teile der Bevölkerung. Denn die Bereitschaft zum Opfer-Bringen ist seit langem eines der prägendsten Merkmale zahlreicher HELD*INNEN: KRIEGERHELDEN wird etwa zugeschrieben, im Kampf ihr Leben zu geben, um die Gemeinschaft zu retten und auch den zivilen ALLTAGSHELDEN wird attestiert, Opfer zu bringen, wenn sie im Zuge ihres Engagements auf Freizeit verzichten, mehr tun, als sie tun müssen, unzählige Stunden ehrenamtlicher Arbeit verrichten sowie die Gesundheit oder das eigene Leben riskieren (cf. Koch 2020: 56-74; 145-152). Aufgrund der engen Verbindung zwischen Opferbereitschaft und HELDENTUM finden sich auch in zahlreichen Darstellungen von CORONA-HELD*INNEN derartige Zuschreibungen. Oft wird dabei angeführt, die Opfer von CORONA-HELD*INNEN würde anderen zugutekommen. Mit einer Toposanalyse (Wengeler 2003, 2007, 2017) können derartige Aussagen eingehender untersuchen werden. Dies kann insbesondere für kulturwissenschaftliche Fragestellungen wichtige Erkenntnisse liefern, da Topoi habituelle Argumentationsmuster sind, die sich im Gebrauch sowohl verfestigen als auch modifiziert werden können (cf. Wengeler 2017: 268f.). Daher eröffnet ihre Analyse Einsichten „über typische, wichtige oder dominante Denkweisen, Sichtweisen, Wahrnehmungsmuster bestimmter Gruppen, in einem bestimmten Zeitraum, bezogen auf ein bestimmtes Thema“" (ibd.: 269). Eines der in den Heroisierungen am häufigsten vorkommenden Argumentationsmuster kann als Topos von der Opferbereitschaft beschrieben werden, der besagt: Weil ein Mensch 
bereit ist, Opfer zu bringen (Freizeit, Kraft, Geld, Luxus, Gesundheit), profitiert die Gesellschaft davon.

Diese Argumentationsstruktur liegt etwa den folgenden Aussagen zugrunde:

Sie setzen ihre Gesundheit aufs Spiel um Infizierten zu helfen und die Pandemie einzudämmen. Sie sorgen dafür, dass unsere Supermärkte gefüllt sind und keine Massenpanik ausbricht. Sie sind die stillen Helden der Coronakrise.

(Clemens 2020, Hervorheb. D. K.)

Der stehende Applaus diese Woche im Bundestag galt vor allem ihnen: Unseren Corona-Helden in der Alten- und Krankenpflege. Sie tragen zusätzlich zur Arbeitsbelastung nun noch das Risiko einer Infizierung, weil Schutzausrüstung fehlt.

(Link 2020, Hervorheb. D. K.)

Für ihren Job als Krankenschwester verzichtet Christina Knappich auf die Nähe zu ihrer Familie. [...] ,Wir bereiten uns hier im Krankenhaus gerade alle für den Notfall vor. Ich habe meinen Mann schon gewarnt, dass es jetzt oft sehr spät werden kann, ehe ich nach Hause komme. Ich rechne mit vielen Überstunden, aber ich bin stolz auf meine Arbeit und mache sie gerne, weil ich helfen kann.“

(Bild.de 2020a, Hervorheb. D. K.)

Der Topos von der Opferbereitschaft ist ein weit verbreitetes sprachliches Muster im Heldendiskurs, mit dem aus den Opfern von HELD* INNEN eine positive Zukunftserwartung abgeleitet wird. Innerhalb des Corona-Diskurses wird mit ihm betont, dass insbesondere Menschen in „systemrelevanten“ Berufen durch ihre Opferbereitschaft unsere Gesellschaft am Laufen hielten, wovon wiederum die gesamte Bevölkerung profitiere. Oft geht mit dieser Opferbereitschaft ein hohes persönliches Risiko einher. Denn die CORONA-HELD*INNEN isolieren sich nicht im Home-Office, sondern kommen bei der Ausübung ihrer Berufe zwangsläufig in Kontakt mit zahlreichen Personen und sind daher einer erhöhten Ansteckungsgefahr ausgesetzt. Wie viele tradierte Heldenfiguren zeichnen sich also auch viele CORONA-HELD*INNEN durch ihren heldenhaften Mut und die Bereitschaft aus, für ihre Mitmenschen eine Gefahr auf sich zu nehmen. Aufgrund dessen wird vermehrt angeführt, die CORONA-HELD*INNEN verdienten für ihre Taten den Dank und die Anerkennung der Gesellschaft, so z. B. auf Nachrichtenportalen, die Rubriken zu CORONA-HELD*INNEN unterhalten:

Sie arbeiten rund um die Uhr, begeben sich freiwillig in Kontakt mit Corona-Patienten - und bekommen viel zu selten dafür einen Dank. Hier lernen Sie die Hannoveraner kennen, auf die es derzeit ganz besonders ankommt - stellvertretend für unzählige weitere Helfer in der Region.

(Hannoversche Allgemeine 2020)

Die Heldinnen und Helden der Corona-Pandemie sind Menschen, die vor Ort das Leben weiter möglich machen. Jene Männer und Frauen, die in Krankenhäusern, Apotheken oder an den Supermarkt-Kassen arbeiten, damit unsere Grundversorgung gesichert ist. Homeoffice geht hier nicht. Für diese Menschen ist harte Arbeit mit Kunden-Kontakt im Dienste der Gemeinschaft der beschwerliche Alltag. Dafür wollen wir herzlich DANKE! sagen. An dieser Stelle finden Sie stellvertretend für viele Heldinnen und Helden der Krise in Werne Menschen, bei denen wir uns bedanken möchten.

(Wellerdiek/Witzig 2020) 
Hier wird abermals auf die positiven Effekte der Risiko- und Opferbereitschaft von CoRONAHELD*INNEN verwiesen und die Konsequenz gezogen, dass ihnen die Gesellschaft dafür Dank und Anerkennung schulde. Dieser kausale Schluss basiert auf dem Ehrungstopos, der besagt: Weil Menschen mit ihren Taten die Gesellschaft besser gemacht haben, verdienen sie Dank und Anerkennung, weshalb wir sie öffentlich ehren, bzw. ihrer gedenken.

Für die Heldwerdung der CORONA-HELD*INNEN ist insbesondere die Kombination des Topos von der Opferbereitschaft und des Ehrungstopos von besonderer Relevanz. Denn „,normale“ Menschen werden innerhalb von Einsetzungsriten zu HELD*INNEN erhoben (cf. Jentges 2010: 80). Diese Riten beinhalten oft einen ,symbolischen Tausch“ (Flaig 2009; Koch 2020: 153159. Dieser Tausch besteht im Wesentlichen aus zwei Komponenten: Erstens bringt der HELD oder die HELDIN im Zuge seines/ihres Dienstes an der Gemeinschaft ein Opfer, wofür ihm/ihr zweitens die Gesellschaft Dank und Anerkennung bekundet. Diese beiden Elemente verdichten sich im Gebrauch des Topos von der Opferbereitschaft und des Ehrungstopos. Diese Akte des symbolischen Tausches zeigen, dass HELD*INNEN oft innerhalb tradierter ritueller Strukturen heroisiert werden.

\section{Wie anonyme Personen(-gruppen) kollektiv zu Held*innen erhoben werden}

Anders als in der Vergangenheit werden heute jedoch seltener Einzelpersonen zu HELD*INNEN erhoben, sondern vermehrt der abstrakte Heldentypus der namenlosen CORONA-HELD*INNEN. Eine derartige Ausrichtung ist auch in den oben aufgeführten Beispielen zu verzeichnen („Hier lernen Sie die Hannoveraner kennen, auf die es derzeit ganz besonders ankommt - stellvertretend für unzählige weitere Helfer in der Region“; „An dieser Stelle finden Sie stellvertretend für viele Heldinnen und Helden der Krise in Werne Menschen, bei denen wir uns bedanken möchten."). Auch diese Aussagen basieren auf einem im Heldendiskurs etablierten Argumentationsmuster. Hierbei handelt es sich um den Topos vom stellvertretenden Dank, der besagt: Weil nicht jeder für seinen Dienst an der Gesellschaft ausgezeichnet werden kann, werden einige Wenige stellvertretend für alle Engagierten geehrt.

Dieser Topos findet sich häufig in Heroisierungen von ALLTAGSHELD*INNEN, in denen er wesentlich zum Abbau klassischer Beschränkungen zur Erlangung des Heldenstatus und der Verlagerung von HELDENTUM ins Alltägliche beigetragen hat. Denn wenn schon kleine Akte der alltäglichen Hilfeleistung als Heldentaten anerkannt werden, wird HELDENTUM zu einem Massenphänomen. Dies hat zur Folge, dass nicht allen ALLTAGSHELD*INNEN die ihnen zustehende Anerkennung entgegengebracht werden kann. Daher wird durch sprachliche Muster wie dem Topos vom stellvertretenden Dank pauschalisierend allen Personen der Heldenstatus zugesprochen, die ähnlich handeln wie die in dem jeweiligen Kontext heroisierten ALLTAGSHELD*INNEN. Auf diese Weise wird der Heldenstatus von konkreten Personen entkoppelt und auf ein Rollenmuster verlagert. Dieser Trend zur Entpersonalisierung von HELDENTUM wird auch durch bestimmte Darstellungsformen auf der bildlichen Ebene befördert. Insbesondere Unternehmen nutzen diese, um ihren Angestellten während der Corona-Pandemie kollektiv einen Heldenstatus zuzusprechen. Auf Facebook und Instagram teilen etwa die Einzelhändler Netto und Edeka sowie der Nordhessische Verkehrsverbund Anzeigen, in denen sie ihre Angestellten als „Helden“ bezeichnen und ihnen für ihre Arbeit danken: 


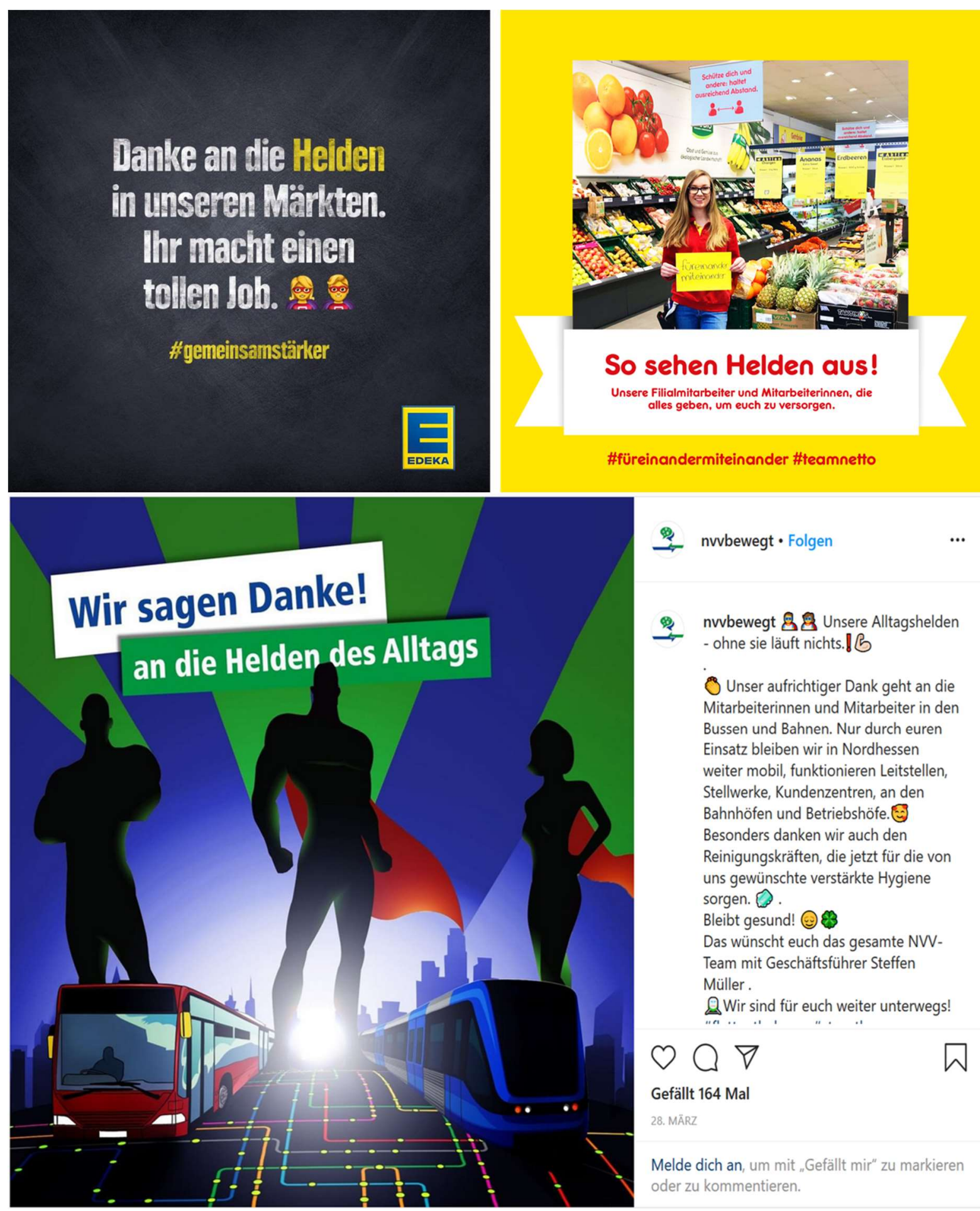

Abbildung 2: Der Gebrauch einer Superheldenikonografie in Heroisierungen von Corona-Held*innen (Netto Marken-Discount 2020; Edeka 2020; nvvbewegt 2020) ${ }^{2}$

In diesen Darstellungen werden keine konkreten Personen heroisiert, sondern verallgemeinernd der Typus der AlltagShELD*IN in Zeiten der Corona-Pandemie. Ähnlich wie in rein sprachlich-textuellen Heroisierungen, die sich des Topos vom stellvertretenden Dank bedienen, fungieren die abgebildeten HELD*INNEN auch hier lediglich als „Platzhalter“ für eine Vielzahl von Personen, die auf diese Weise mitheroisiert werden. So steht die namenlose Frau innerhalb der

2 Die Bilder sind öffentlichen und frei zugänglichen Werbekampagnen entnommen. 
Anzeige von Netto stellvertretend für alle Mitarbeiter*innen des Unternehmens (,,So sehen Helden aus! Unsere Filialmitarbeiter und Mitarbeiterinnen, die alles geben, um euch zu versorgen."). Auch in den Anzeigen von Edeka und dem NVV wird nicht konkreten Personen gedankt, sondern allen „Helden in unseren Märkten“ bzw. ,unsere[n] Alltagshelden [...] in den Bussen und Bahnen“. Diese beiden Heroisierungen zeichnen sich zudem durch ihre Referenzen auf Superheld*innen aus: Die Mitarbeiter*innen in der Edeka-Anzeige werden durch Superheld*innen-Emojis symbolisiert und die des Nordhessischen Verkehrsverbunds durch Schemen von Superheld*innen, in beiden Fällen gut zu erkennen an den roten Umhängen. Derartige Formen der Darstellung haben in den letzten Jahren massiv an Bedeutung im Heldendiskurs gewonnen und zur Etablierung einer Superheldenikonografie beigetragen (cf. Koch 2020: 261274). Diese wird heute in Heroisierungen verschiedenster Personen genutzt. Da sich Bilder durch ihre semantische Offenheit auszeichnen, ist das ,heldische“ Vorwissen für die Interpretation derartiger Darstellungen von großer Bedeutung. Zu diesem Wissen gehört etwa, dass sich Superheld*innen typischerweise für das Wohl der Gemeinschaft einsetzen und damit die Welt zu einem besseren Ort machen. Auch verzichten sie auf das Zusammensein mit ihrer Familie und stellen ihre eigenen Bedürfnisse zugunsten der Allgemeinheit hintan. Dabei streben sie nicht nach Ruhm, sondern agieren meistens in der Anonymität. Diese Wissensbestände haben sich durch die zunehmende Präsenz von Superhelden*innen in der Populärkultur in weiten Teilen der Bevölkerung etabliert. Insbesondere die in den letzten Jahren sehr erfolgreichen Superhelden-Filme des Marvel Cinematic Universe und des DC-Universums haben maßgeblich hierzu beigetragen. Auch zahlreiche ALLTAGSHELD*INNEN zeichnen sich durch derartige Charakteristika aus, was sie mit Superheld*innen verbindet. Durch den Gebrauch von Elementen der Superheldenikonografie wird implizit auf diese Parallelen zwischen Menschen, die sich im Alltag für ihre Mitmenschen einsetzen, und Superheld*innen verwiesen und die Rezipient*innen so aufgefordert, ALLTAGSHELD*INNEN einen Heldenstatus zuzuschreiben. Diese interpretationslenkenden Referenzen auf Superheld*innen sind Schlagbilder und als solche ein.

in Diskursen häufig vorkommendes und bekanntes Bildmotiv, das einen zentralen ,Schlüssel' zu Deutungsräumen bietet und so das Textverstehen maßgeblich beeinflusst. [...] So sind sie stark kontextdeterminiert, haben einen programmatischen Gehalt und einen herausgehobenen Status in der öffentlichen Kommunikation, verkürzen komplexe Argumentationen, markieren und grenzen Ideologien ab und leiten zum Handeln an.

(Diekmannshenke 2011: 159)

Wie das Hochwertwort Held halten uns also auch bildliche Darstellungen von HELD*INNEN implizit dazu an, ihnen positiv gegenüberzustehen, zu ihnen aufzublicken und ihrem Beispiel nachzueifern. Aufgrund der deontischen Bedeutungen dieser Schlagbilder und deren Popularität werden sie von Unternehmen genutzt, um dem Teil der Belegschaft zu danken, der nicht im Homeoffice arbeitet und sich daher schneller mit dem Virus anstecken kann. Oft werden dabei Berufsgruppen heroisiert, die allgemein nur wenig gesellschaftliches Ansehen genießen (z. B. Angestellte im Supermarkt, LKW-Fahrer*innen, Pfleger*innen). Diese Form der Würdigung kann sich sowohl positiv auf die Außenwahrnehmung als auch auf das Selbstbild der heroisierten Arbeitnehmer*innen auswirken. Da derartige Heroisierungen die Arbeitsmoral verbessern können, schreiben manche Firmen ihrer gesamten Belegschaft einen Heldenstatus zu, auch 
wenn diese keinem erhöhten Infektionsrisiko ausgesetzt ist. So stellte etwa der Verlag Copernicus zu Beginn der Pandemie in einer Reihe von internen Newslettern heraus, dass alle Angestellten „Helden des Alltags“ seien:

Tja, mit dieser Serie habt ihr gesehen, dass nun wirklich JEDER Copernicaner auf seine Weise ein Held des Alltags ist. [...] Unsere Firma ist ein wirklich Superspitzenteam und ich danke euch 1000fach für euren unermüdlichen Einsatz trotz aller Widrigkeiten, die es aktuell gibt!

(Rasmussen 2020)

\section{Was kommt nach der öffentlichen Ehrung von Corona-Held*innen?}

Trotz derartiger Tendenzen liegt der öffentliche Fokus auf CORONA-HELD*INNEN in ,systemrelevanten“ Berufen. Mit diesen so unverzichtbaren HELD*INNEN rücken jedoch auch deren allzu oft prekäre Beschäftigungsverhältnisse in das Bewusstsein der Gesellschaft und es wird darüber diskutiert, ob sie nicht vielleicht mehr verdienten als nur öffentliche Dankesbekundungen:

Nach Applaus von den Balkonen und vielerlei Dankesbekundungen nun auch bares Geld für die „Helden“ der Coronakrise: Der Berliner Senat hat Krankenschwestern, Polizisten und Kassiererinnen in den Supermärkten einen Bonus in Aussicht gestellt.

(n-tv 2020)

Nun, in der Corona-Krise, wächst in der Bevölkerung das Bewusstsein für die Leistungen dieser Berufsgruppen. Auf den Balkonen wird geklatscht für die „Corona-Helden“. Und in der Politik werden Forderungen laut, zum Beispiel nach Sonderzahlungen, die am besten steuerfrei sein sollen.

(Liebold 2020)

Bisher wurde nur selten diskutiert, ob HELD*INNEN für ihre Taten nicht nur ideellen Dank, sondern auch eine materielle Vergütung verdienten. Es handelt sich hierbei also um ein kommunikatives Muster, welches die Heroisierungen von CORONA-HELD*INNEN von denen der meisten anderen HELD*INNEN unterscheidet. Mit der Forderung nach Boni oder anderen Vergütungen ist das Ansinnen verbunden, den CORONA-HELD*INNEN etwas wiederzugegeben, wovon sie im Alltag konkret profitieren. Auf diese Weise könne sich die Gesellschaft für das Engagement von Menschen aus ,systemrelevanten“ Berufen revanchieren. Daneben deuten einige Maßnahmen, wie das oben thematisierte Stipendium für „Corona-Helden“, darauf hin, dass durch materielle Vergütungen auch ein zusätzlicher Anreiz geschaffen werden soll, sich zum Wohle der Gemeinschaft einzusetzen und zur CORONA-HELD*IN zu werden. Paradoxerweise könnte dieses Ansinnen genau das Gegenteil bewirken. Denn wenn HeLDENTUM „bezahlt“ wird, ist heldenhaftes Handeln kein freiwilliges Opfer an die Gemeinschaft mehr. Der Nimbus des HeroISCHEN könnte verloren gehen und der Heldenstatus an Strahlkraft einbüßen. Ganz unbestreitbar haben HELD*INNEN gerade in Krisenzeiten einen „Wert“ für die Gesellschaft. Doch ob sich dieser in monetären Beträgen ausdrücken lässt, bleibt abzuwarten. 
Und ob die Bevölkerung immer noch klatscht für die „Corona-Helden“, wenn sie für deren Besserstellung ins eigene Portemonnaie greifen muss. [...] Noch sind die ökonomischen Schäden der Corona-Pandemie nicht absehbar. Und was aus den Altenpflegerinnen, LKW-Fahrern und Kassiererinnen wird, die gerade so sehr gebraucht werden. Aber je länger die Krise dauert, desto mehr Unternehmen könnten pleite gehen und viele Menschen würden ihre Jobs verlieren. Erst dann wird sich zeigen, wie die Gesellschaft wirklich zu ihren Corona-Helden steht. Ob es nur beim Applaus bleibt oder sie in Zukunft auch mehr Geld bekommen.

(Liebold 2020)

\section{$5 \quad$ Fazit}

Festzuhalten ist, dass seit Beginn der Corona-Pandemie zahlreiche Menschen zu „Helden“ erhoben werden. Diese CORONA-HELD*INNEN tragen mit ihren Heldentaten maßgeblich zur Überwindung der Krise bei. Gleichzeitig sind sie gesellschaftliche Leitfiguren und leben uns vor, welche Werte und Verhaltensweisen im „Kampf“ gegen das Virus ,zum Sieg führen“ (können). Die Heroisierungen von CORONA-HELD*INNEN erfüllen damit vor allem zwei Funktionen: Zum einen dienen sie zur Vermittlung solidarischer Werte und leiten zum gemeinschaftlichen Handeln an. Zum anderen sind sie eine Möglichkeit, engagierte Menschen in das öffentliche Bewusstsein zu rücken, ihnen Anerkennung entgegenzubringen und ihnen zu danken.

Zwar handelt es sich bei der umfangreichen Heroisierungspraxis von CORONA-HELD*INNEN um ein Krisenphänomen, jedoch wird dabei kein gänzlich neuer Heldentypus erschaffen. Vielmehr sind CORONA-HELD*INNEN eine spezielle Ausprägung der seit einigen Jahren sehr präsenten Alltagsheld*InNen. Daher teilen Alltags- und Corona-HelD*INNEN zahlreiche Merkmale miteinander: Ihr HELDENTUM wird im Alltag verortet, weshalb schon kleine Akte der Hilfeleistung als Heldentaten anerkannt werden. Diese Ausrichtung eröffnet potenziell jedem oder jeder HELD oder HELDIN zu werden, unabhängig seines oder ihres Berufes oder Geschlechts. Zusätzlich zu diesen inhaltlichen Überschneidungen werden in den Heroisierungen beider Heldentypen oft dieselben kommunikativen Muster verwendet. So wird häufig angeführt, CORONA-HELD*INNEN ,halten die Gesellschaft am Laufen“, worin sich das im Alltagsheldendiskurs etablierte Motiv des die-Welt-im-Kleinen-besser-Machens manifestiert. Auch die Darstellungen ihrer Heldentaten folgen konventionalisierten sprachlichen Mustern: Diese lassen sich häufig unter das semantische Feld „Hilfe/Engagement“ subsummieren. Oft folgt hierauf die Präposition für und die Nennung einer hilfsbedürftigen Gruppe (,,sie setzen sich für ihre Mitmenschen ein"). Insbesondere in dieser syntagmatischen Struktur spiegelt sich die enge Verbindung zwischen den HELD*INNEN und der Gesellschaft wider. Auf diese Weise - und durch die häufige Verwendung von Wörtern des semantischen Feldes „Gemeinschaft“ - werden die CORONA-HELD*INNEN als zutiefst solidarisch und gemeinschaftlich orientierte Personen charakterisiert.

Qua der deontischen Bedeutung des Hochwertwortes Held und ihres daraus resultierenden Status als gesellschaftliche Leitfiguren fordern uns die CORONA-HELD*INNEN auf, ihrem Beispiel nachzueifern und damit selbst zur HELD*IN zu werden. Da potenziell jede*r in der Krise HELD*IN werden kann, werden nur selten Einzelpersonen heroisiert, sondern primär der abstrakt-ideelle Typus der CORONA-HELD*INNEN. Konkrete Personen fungieren dann oft lediglich als „Platzhalter“ für alle anderen Bürger*innen, die sich auf ähnliche Art und Weise verdient machen. Auf kommunikativer Ebene spiegelt sich diese Entpersonalisierung von HELDENTUM 
im häufigen Gebrauch des Topos vom stellvertretenden Dank und der Nutzung von Elementen einer Superheldenikonografie.

Der Heldenstatus von CORONA-HELD*INNEN ist derzeit weitgehend unhinterfragt. Sie werden als ,echte Helden“ wahrgenommen, da sich in ihnen Merkmale von zivil-demokratischen und tradiert-kriegerischen Heldenkonzepten verdichten. Hierdurch fügen sie sich in das „Heldenwissen" eines großen Teils der Bevölkerung ein. Insbesondere in Heroisierungen von CORONAHELD*INNEN aus medizinischen Berufen werden oft Wörter des semantischen Feldes „Militärischer Kampf" genutzt und diese damit als Kämpfer*innen konzeptualisiert. Auf diese Weise werden sie nicht nur in Tradition zu klassischen KRIEGERHELDEN gestellt, sondern gleichzeitig wird so auch eine bestimmte Perspektive auf die Wirklichkeit vorgegeben: Wenn die Begegnung mit dem Virus ein „Kampf“ ist, dann kann dieser mit den richtigen Maßnahmen und Anstrengungen gewonnen werden. Die Gesellschaft schreibt sich durch diese Konzeptualisierungspraxis also Handlungsmacht und Einfluss auf eine Situation zu, die ihr in Zeiten der Krise abhandengekommen ist. Dieses Gefühl von vermeintlicher Kontrolle wird durch den häufigen Gebrauch des Topos von der Opferbereitschaft zusätzlich befördert, da mit diesem Argumentationsmuster aus den Opfern und Entbehrungen der CORONA-HELD*INNEN ein positiver Effekt auf die Gesellschaft abgeleitet wird.

Die Analysen dieses Beitrags zeigen, dass HELD*INNEN zutiefst eingebettet sind in die bestehenden Werte und Normen einer Gesellschaft und dass Heldenbilder auf aktuelle Veränderungen reagieren. Trotz dieses Anpassungsprozesses an aktuelle Gegebenheiten weisen die Muster der multimodalen Konstitution von HELDENTUM jedoch ein hohes Maß an Beständigkeit auf. Wie einleitend angeführt wurde, liefert die Untersuchung von HELD*INNEN neben derartigen linguistischen Erkenntnissen auch Einsichten über die Beschaffenheit unserer Gesellschaft und unseres Umgangs mit Krisen. Vor diesem Hintergrund ist festzuhalten, dass zahlreiche Heroisierungen von CORONA-HELD*INNEN auf die Vermittlung eines gesamtgesellschaftlichen Gemeinschaftsgefühls abzielen. Hierin spiegelt sich die Vorstellung wider, dass immense Herausforderungen nur dann bewältigt werden können, wenn die gesamte Bevölkerung sich als Teil eines großen Ganzen versteht und individuelle Bedürfnisse zugunsten der Mehrheit zurückgesteckt werden. Die Heroisierung von CORONA-HELD*INNEN und die Umdeutung des passiven Leidens unter der Krankheit zu einem aktiven „Kampf gegen den unsichtbaren Feind“ schwört die Bevölkerung zudem auf ein gemeinsames Ziel ein. Durch diese Fokussierung werden Ressourcen und Anstrengungen gebündelt, was sich wiederum positiv auf den Ausgang der Krise auswirken kann. Denn die autosuggestive Zuschreibung von Handlungsmacht kann dazu beitragen, dass die Gesellschaft auch in einer kritischen Situation weitestgehend rational handelt. Dass sich bisher die Mehrheit der Deutschen an die Vorgaben der Politik hält und Ausgangsbeschränkungen akzeptiert, könnte vor diesem Hintergrund auch von den zahlreichen Heroisierungen von CORONA-HELD*INNEN (aus dem medizinischen Sektor) befördert worden sein.

Es wird deutlich, dass „Helden“ aufgrund der „wirklichkeitskonstituierenden Kraft der Sprache“ (Gardt 2018: 1) sowohl unseren Blick auf eine Krise als auch unser konkretes Handeln in dieser Ausnahmesituation maßgeblich beeinflussen können. Daher sind „Helden“ während der Corona-Pandemie von besonderer Bedeutung. Denn sie fungieren als Vorbilder und vermitteln Werte und Normen. Sie entfalten eine integrative Wirkung, stiften Orientierung sowie Identität und können politische Maßnahmen legitimieren. Diese Politisierung von HELD*INNEN lässt 
sich gerade auch in jüngster Zeit beobachten, wenn die Forderung nach einer finanziellen Besserstellung von Menschen in „systemrelevanten“ Berufen mit Verweisen auf deren besondere Leistungen und ihrer heldenhaften Opferbereitschaft untermauert wird. Zu einer ,postheroischen Gesellschaft" passt dies nicht. Hält man sich die aktuelle Superheldenikonografie in unserer kommunikativen und kulturellen Wirklichkeit vor Augen, so liegt es vielmehr nahe, von einer „superheroischen Gesellschaft“ zu sprechen (cf. hierzu auch Koch 2020: 305-335).

\section{Literaturverzeichnis}

1. FC Nürnberg (2020): „FCN-Ehrentrikot für Helden in Krankenhäusern und Supermärkten“. fcn.de/news/artikel/fcn-ehrentrikot-fuer-helden-in-krankenhaeusern-und-supermaerkten/ [03.04.2020].

Bennet, Brian/Berenson, Tessa (2020): “'Our Big War’ As Coronavirus Spreads, Trump Refashions Himself as a Wartime President". time.com/5806657/donald-trump-coronaviruswar-china/ [14.04.2020].

Bild.de (2020a): „Die Coronahelden - Diese Menschen halten Deutschland am Laufen“. bild.de/news/inland/news-inland/die-coronahelden-diese-menschen-halten-deutschlandam-laufen-69542128.bild.html [30.03.2020].

Bild.de (2020b): „Steuer: Finanzminister Scholz denkt über Bonus für Corona-Helden nach“. bild.de/politik/inland/politik-inland/steuer-finanzminister-scholz-denkt-ueber-bonus-fuercorona-helden-nach-69595076.bild.html [07.04.2020].

Bundesministerium der Finanzen (2020): „Kampf gegen Corona: Größtes Hilfspaket in der Geschichte Deutschlands." bundesfinanzministerium.de/Content/DE/Standardartikel/Themen/Schlaglichter/Corona-Schutzschild/2020-03-13-Milliarden-Schutzschild-fuer-

Deutschland.html [14.04.2020].

Clemens, Wergin (2020): Corona: Die Helden des Krisenalltags verdienen unseren Dank. welt.de/debatte/kommentare/article206645409/Corona-Die-Helden-des-Krisenalltags-verdienen-unseren-Dank.html [07.04.2020].

Diekmannshenke, Hajo (2011): „,Schlagbilder‘. Diskursanalyse politischer Schlüsselbilder“. In: Diekmannshenke, Hajo/Klemm, Michael/Stöckl, Hartmut (eds.): Bildlinguistik: Theorien - Methoden - Fallbeispiele. Berlin, Schmidt: 159-184. (= Philologische Studien und Quellen 228).

Edeka (2020): Danke an die Helden in unseren Märkten. facebook.com/EDEKA/photos/a.204899552919220/2829232513819231/?type=3\&refid $=52 \& \_$tn_ $=\mathrm{EH}-\mathrm{R}$ [30.03.2020].

Einkaufshelden-Team (2020): Einkaufshelden für Sie in Staufenberg unterwegs. Flyer, verteilt vor einem Supermarkt in Staufenberg im März/April 2020.

Felder, Ekkehard (2009): „Das Forschungsnetzwerk Sprache und Wissen - Zielsetzungen und Inhalte“. In: Felder, Ekkehard Felder/Müller, Marcus (eds.): Sprache und Wissen. Theorie, Praxis und Erkenntnisinteresse des Forschungsnetzwerkes ,,Sprache und Wissen“. Berlin/ New York, de Gruyter: 11-18. (= Sprache und Wissen 3).

Flaig, Egon (2009): „Symbolischer Tausch und heldischer Tod.“ In: Merkur: Deutsche Zeitschrift für europäisches Denken 63 (724/725): 843-848.

focus.de: „Nur 2000 Euro im Monat - brutto: So wenig verdienen unsere Corona-Helden“. focus.de/finanzen/karriere/berufsleben/gehalt/gehaltscheck-fuer-systemrelevante-berufe- 
nur-2000-euro-im-monat-brutto-so-wenig-verdienen-unsere-corona-helden_id_11820059. html [30.03.2020].

Gardt, Andreas (2012): „Textsemantik. Methoden der Bedeutungserschließung“. In: Bär, Jochen A./Müller, Marcus (eds.): Geschichte der Sprache-Sprache der Geschichte. Probleme und Perspektiven der historischen Sprachwissenschaft des Deutschen. Oskar Reichmann zum 75. Geburtstag. Berlin, Akademie-Verlag: 15-37. (=Lingua Historica Germanica 3).

Gardt, Andreas (2018): „Wort und Welt. Konstruktivismus und Realismus in der Sprachtheorie“. In: Felder, Ekkehard/Gardt, Andreas (eds.): Wirklichkeit oder Konstruktion? Sprachtheoretische und interdisziplinäre Aspekte einer brisanten Alternative. Berlin/Boston, de Gruyter: 1-44.

Hannoversche Allgemeine (2020): „Das sind Hannovers Helden in der Corona-Krise“. haz.de/Hannover/Aus-der-Stadt/Das-sind-Hannovers-Helden-in-der-Corona-Krise [30.03.2020].

Hermanns, Fritz (2012): „Sprachgeschichte als Mentalitätsgeschichte“. In: Hermanns, Fritz et al. (eds.): Der Sitz der Sprache im Leben. Beiträge zu einer kulturanalytischen Linguistik. Berlin, de Gruyter: 5-36.

Jentges, Erik (2010): Die soziale Magie politischer Repräsentation. Charisma und Anerkennung in der Zivilgesellschaft. Bielefeld: transcript (= Sozialtheorie).

Klug, Nina-Maria/Stöckl, Hartmut (2015): „Sprache im multimodalen Kontext““. In: Felder, Ekkehard/Gardt, Andreas (eds.): Handbuch Sprache und Wissen. Berlin/Boston, de Gruyter: 242-264. (= Handbücher Sprachwissen 1).

Koch, Daniel (2020): Der „Held“ im Deutschen. Eine linguistische Konzeptanalyse. Berlin: de Gruyter (= Sprache und Wissen (SuW) 43).

Lidl Deutschland (2020): \#lidlsuperhelden. facebook.com/story.php?story_ $\mathrm{fbid}=10158172018737258 \&$ substory_index $=0 \& \mathrm{id}=278565202257$ [30.03.2020].

Liebold, Jan (2020): „Systemrelevante Berufe: Welchen Wert haben Helden?“ tagesschau.de/inland/bab-helden-101.html [06.04.2020].

Link, Albert (2020): „Mehr als warme Worte für unsere Corona-Helden!“‘ bild.de/politik/kolumnen/kolumne/standpunkt-mehr-als-warme-worte-fuer-unsere-corona-helden69693904.bild.html [30.03.2020].

Lukesch, Andreas (2020): „Corona und die Folgen. Damit die Helden Helden bleiben“. In: HNA Hessisch/Niedersächsische Allgemeine, 11.04.2020: 5.

Mundt, Anna (2020): „Coronakrise in Deutschland: Sie sind Helden des Alltags“. Tagesschau.de, 26.03.2020. tagesschau.de/inland/helden-des-alltags-corona-101.html [30.03.2020].

Münkler, Herfried (2006): Der Wandel des Krieges. Von der Symmetrie zur Asymmetrie. Weilerswist: Velbrück.

Münkler, Herfried (2007): „Heroische und postheroische Gesellschaften“. In: Merkur: Deutsche Zeitschrift für europäisches Denken 61, 8/9: 742-752.

Münkler, Herfried (2015): Kriegssplitter. Die Evolution der Gewalt im 20. und 21. Jahrhundert. Berlin: Rowohlt.

myStipendium.de (2020): „Alltagshelden-Stipendium“. mystipendium.de/stipendien/alltagshelden-stipendium [27.04.2020]. 
n-tv (2020): „Berlin stellt Corona-Helden Bonus in Aussicht“. n-tv.de/politik/Berlin-stelltCorona-Helden-Bonus-in-Aussicht-article21671560.html [30.03.2020].

Netto Marken-Discount (2020): „So sehen Helden aus!“ facebook.com/nettomarkendiscount/photos/a.1714593052086028/2506122932933032/?type $=3 \&$ refid $=52 \&$ th $=$ EH$\mathrm{R}$ [01.04.2020].

nvvbewegt (2020): „Wir sagen Danke! an die Helden des Alltags“. instagram.com/p/BRDIM315Oy/?igshid=196g2wi9c3kpf [17.04.2020]

Rasmussen, Martin (2020): Tag 19: Klingelingeling, Hier kommt der Eiermann... Interner Newsletter der Firma Copernicus, Göttingen. (Persönliche Kommunikation).

Späth, Sebastian (2020a): „Corona-Alltags-Heldin: Tamara Gabel, 26, Apothekerin, stellt nun selbst Desinfektionsmittel her". spiegel.de/panorama/gesellschaft/corona-alltags-heldtamara-gabel-26-apothekerin-stellt-nun-selbst-desinfektionsmittel-her-a-49a86cbf-bfc24849-8652-c99bd06e8963 [01.04.2020].

Späth, Sebastian (2020b): „Corona-Alltags-Held: Jakob Stuhlfelder, 25, Zahnarzt, betreut weiter seine Patienten“. spiegel.de/panorama/gesellschaft/corona-held-jakob-stuhlfelder-25zahnarzt-betreut-weiter-seine-patienten-a-33fe08fe-46d1-4cd3-8977-3a5c25b812cd [30.03.2020].

spiegel.de (2020a): „'Wir bleiben für euch hier. Bleibt bitte für uns daheim““. spiegel.de/gesundheit/coronavirus-aufhalten-wir-bleiben-fuer-euch-hier-bleibt-bitte-fuer-uns-daheim-a10ea79a3-3474-4af7-a019-8d543e751d6f [19.03.2020].

spiegel.de (2020b): „Heldinnen und Helden des Corona-Alltags“. spiegel.de/thema/heldinnenund-helden-des-corona-alltags/ [30.03.2020].

Steinmeier, Frank-Walter (2020): „Nur der Verzicht verhindert, dass wir dauerhaft verlieren, was wir lieben. " bundespraesident.de/SharedDocs/Reden/DE/Frank-Walter-Steinmeier/Reden/2020/03/200326-Videobotschaft-Coronahelden.html;jsessionid=CA2A214A109A0695 D1D05AF77AE65CA2.2_cid371 [30.03.2020].

STIMME.de (2020): „Helden des Alltags: Sie halten das System am Laufen“. stimme.de/heilbronn/nachrichten/region/Helden-des-Alltags-Sie-halten-das-System-am-Laufen;art1408 97,4343538 [15.04.2020].

Süddeutsche Zeitung Online (2020): „Frankreich im ,Gesundheitskrieg' gegen Corona“. sueddeutsche.de/gesundheit/gesundheit-frankreich-im-gesundheitskrieg-gegen-corona-dpa.urnnewsml-dpa-com-20090101-200316-99-353511 [14.04.2020].

Töpper, Verena (2020): „Corona-Alltags-Held: Uwe Kleinsorge, 44, Lastwagenfahrer, steht jetzt oft vor verschlossenen Toiletten“. spiegel.de/karriere/corona-alltags-held-lastwagenfahrer-erzaehlt-aus-seinem-berufsalltag-a-5adb3f9b-072f-4a16-a3b6-35e204556408 [09.04.2020].

Wellerdiek, Andrea/Witzig, Silvia (2020): „Unsere Aktion für Heldinnen und Helden in Werne in der Corona-Krise“. ruhrnachrichten.de/werne/wir-sagen-danke-an-die-helden-dercorona-krise-diese-menschen-halten-in-werne-alles-am-laufen-1507138.html [30.03.2020].

Wengeler, Martin (2003): Topos und Diskurs. Begründung einer argumentationsanalytischen Methode und ihre Anwendung auf den Migrationsdiskurs (1960-1985). Tübingen: Niemeyer (= Germanistische Linguistik 244).

Wengeler, Martin (2007): „Topos und Diskurs - Möglichkeiten und Grenzen der topologischen Analyse gesellschaftlicher Debatten“. In: Warnke, Ingo (ed.): Diskurslinguistik nach 
Foucault. Theorie und Gegenstände. Berlin/New York, de Gruyter: 165-186. (=Linguistik, Impulse \& Tendenzen 25).

Wengeler, Martin (2017): „Diskursorientierte Argumentationsanalyse“. In: Niehr, Thomas/Kilian, Jörg/Wengeler, Martin (eds.): Handbuch Sprache und Politik. Band 1. Bremen, Hempen: 261-281. (= Sprache-Politik-Gesellschaft 21.1).

Witterauf, Stefanie (2020): „Corona-Alltags-Heldin: Susanne Rudwill, 56, Kassiererin, will nicht hinter einer Maske lächeln“. spiegel.de/panorama/gesellschaft/corona-alltags-heldinsusanne-rudwill-56-kassiererin-will-nicht-hinter-einer-maske-laecheln-a-721 fe6ea-feab4f3d-a8bc-f3171f9c621b [14.04.2020].

Würth, Wolfgang (2020): „Rentenexperte: Grundrente als Dank an Corona-Helden“. onetz.de/deutschland-welt/weiden-oberpfalz/rentenexperte-grundrente-dank-corona-heldenid3001333.html [30.03.2020].

Würzer, Julian (2020): „Coronavirus in Berlin: Diese Alltags-Helden halten Berlin am Laufen“. morgenpost.de/berlin/article228900661/Corona-Helden-Sie-halten-Berlin-am-Laufen.html [15.04.2020].

ZDF (2020a): „Helden in der Corona-Krise: Apotheker, 28.03.2020“. zdf.de/nachrichten/video/politik-helden-der-krise-apotheker-100.html [30.03.2020]

ZDF (2020b): „37 Grad: Den Laden am Laufen halten“. zdf.de/dokumentation/37-grad/37-denladen-am-laufen-halten-100.html [15.04.2020]. 